\title{
Combined supine and prone imaging acquisition in cardiac SPECT: A turn for the better
}

\author{
Mylan C. Cohen, MD, MPH, MASNC ${ }^{\mathrm{a}, \mathrm{b}, \mathrm{c}, \mathrm{d}}$ \\ a Cardiovascular Informatics, Cardiovascular Service Line, MaineHealth, Portland, ME \\ b Cardiac Imaging and Diagnostics, Maine Medical Center, Portland, ME \\ c Maine Medical Partners MaineHealth Cardiology, Scarborough, ME \\ d Tufts University School of Medicine, Boston, MA
}

Received Dec 20, 2015; accepted Dec 21, 2015 doi: $10.1007 / \mathrm{s} 12350-015-0389-8$

See related article, pp. 1470-1476

\section{INTRODUCTION}

Imagine the following scenario. You are called one afternoon by your colleague in the catheterization laboratory. Your colleague sheepishly informs you that there is good news and bad news about the 65-year-old obese man whom you referred for coronary angiography because of somewhat atypical chest discomfort but a concerning inferior wall defect on pharmacologic hyperemia myocardial single-photon emission-computed tomography (SPECT). The good news is that the patient's coronary arteries are normal. The bad news is that the patient suffered a complication of the procedure that will necessitate observation in the hospital for a day or two. You wonder whether there was anything that could have been done that may have improved the accuracy of the SPECT and obviated the need for the catheterization that resulted in an unnecessary complication. Now imagine that with a simple maneuver, without exposure to additional radiation, without additional cost to the patient, and without any new special equipment in your nuclear cardiology laboratory, you could have reduced that risk by over $50 \%$. Would you have done it?

In this issue of the Journal of Nuclear Cardiology, Taasan and coauthors report the results of a retrospective study of 934 males at a Veterans Administration Medical Center (VAMC) who underwent rest and

Reprint requests: Mylan C. Cohen, MD, MPH, MASNC, Maine Medical Partners MaineHealth Cardiology, 96 Campus Drive, Suite 1, Scarborough, ME 04074; cohenm@mmc.org

J Nucl Cardiol 2016;23:1477-9.

1071-3581/\$34.00

Copyright (C) 2016 American Society of Nuclear Cardiology. regadenoson stress Tc-99 tetrofosmin myocardial perfusion imaging (MPI) with either supine (597 patients) or combined supine and prone image acquisition (337 patients), the laboratory routinely alternating between the two protocols. Combined supine and prone imaging increased diagnostic certainty compared with supineonly imaging, reducing the proportion of equivocal studies from $13 \%$ to $4 \%$ between the two groups $(P<.001)$, most equivocal studies being due to diaphragmatic attenuation. Inferior artifact size was reduced in the combined supine and prone imaging group. The appearance of prone-specific artifacts in the anterior, anteroseptal, and anterolateral walls were "small in size, mild in severity, and easy to recognize." Perhaps the most important findings of the study, however, came from the subset of 116 patients who went on to have coronary angiography within 6 months of SPECT. Accuracy was increased with combined supine and prone imaging compared to supine imaging alone (area under the receiver operating curves: $0.8 \pm 0.06$ vs $0.57 \pm 0.05, P=.004)$ The false-positive rate attributable to inferior wall artifacts in the combined supine and prone imaging group was less than half that of the supine-only imaging group (27\% vs $64 \%$, $P<.001)$. False-positive rates were $40 \%$ higher in supine-only imaging in obese patients compared to $20 \%$ higher in non-obese patients though the difference did not quite reach statistical significance $(P=.06)$, likely related to small sample size in the subsets. ${ }^{1}$

The authors are to be congratulated on this study. Though not the first to demonstrate the benefit of prone imaging in combination with supine imaging, ${ }^{2-11}$ the investigation is unique in that patients were protocoled for either supine-only or combined supine and prone imaging independent of criteria such as obesity or the presence of artifact on supine imaging. Groups were compared rather than patients serving as their own controls. Though it is true that all of the patients were male as a function of the study being performed at a VAMC, which is unique, this might be considered a 
limitation of the study with regard to generalizability. Other limitations included an estimated $20 \%$ rate in failure to prone patients attributable to physical limitations of patients and the coronary angiography correlation study had inherent post-test referral bias.

Knowledge gained from the present report extends that of previous investigators who have shown that patients with inferior wall defects on supine MPI that are not present on prone MPI have a low risk of subsequent cardiac events, similar to that of patients with normal supine-only studies. ${ }^{5}$ Together with quantitative methods, accuracy of combined supine and prone imaging is enhanced, ${ }^{6}$ not only in men but also in women ${ }^{7}$ and obese and non-obese patients. ${ }^{8}$ The technique also increases inter-observer correlation and agreement. ${ }^{9}$

\section{PLACING THE PRESENT STUDY IN CONTEXT: IMPROVING QUALITY}

It is important to consider the present study in the broader context of improving quality in nuclear cardiology laboratories and the value these results potentially represent for SPECT studies performed daily everywhere. Components of a compressive approach to improving quality and being consistent with best practices include - among other things-only performing studies that are appropriate and necessary, employing techniques that reduce radionuclide doses and therefore patients' radiation exposure, and taking steps to improve accuracy of image interpretation. ${ }^{12}$

Artifacts have been a well known and longstanding shortcoming of MPI. The development and wide adoption of gated SPECT represented an advance over planar imaging and yet inferior artifacts remain a limitation of the technique. ${ }^{3}$ Attenuation correction, whether with transmission sources or with low-resolution computed tomography, has been a proven beneficial addition to standard SPECT imaging and is extremely useful in distinguishing true anterior and inferior defects from attenuation artifacts. Nevertheless, the penetration of this technique into most nuclear cardiology laboratories has been low, ${ }^{13}$ in part due to increased expense of the equipment in an era of decreasing MPI volumes and declining reimbursement without additional reimbursement provided for performing this valuable add-on to a standard examination. Many practices, medical centers, and health care systems prefer to hold on to old equipment, utilizing outdated image processing methods.

In a survey in $2013,75 \%$ of nuclear cameras in the United States were 6 years or older, nearly $29 \%$ 11 years or older. ${ }^{14}$ Though not reported, the proportion of nuclear cameras with attenuation correction and iterative reconstruction, relatively new technologies, can be assumed to have been low. In Europe in 2007, attenuation correction was used in only $22 \%$ of studies. ${ }^{13}$ Likewise, adoption of wide beam reconstruction and resolution recovery has likely been low though there are relatively inexpensive software solutions that can be utilized even on older systems. These techniques can be important tools in reducing radiation exposure to patients. ${ }^{15}$ Combined with attenuation correction and newer processing methods, stress-only imaging can substantially reduce radiation exposure as well as duration of studies thereby improving patient comfort, enhancing lab throughput, and increasing the overall efficiency of care that we provide. ${ }^{16,17}$ These technologies and techniques in part drove ASNC's expectation that for the population of patients referred for SPECT or PET MPI, on average a total radiation exposure of $\leq 9 \mathrm{mSv}$ could be achieved in $50 \%$ of studies by 2014 . Whether this goal has actually been met is not entirely certain.

Though prone imaging by itself does not reduce radiation exposure, having greater confidence that an inferior defect is artifactual could result in a higher proportion of stress-only studies being performed on nuclear cameras without attenuation correction. However, despite prone imaging having been described almost 30 years ago, ${ }^{2}$ combined supine and prone imaging is not widely utilized. $^{13}$ As Taason and colleagues correctly point out the benefits of prone imaging include downward displacement of the diaphragm and abdominal organs, compression of anterior chest soft tissue including breast tissue, a shift of the heart more anteriorly, and reduction of patient motion. ${ }^{1}$

There is no doubt that attenuation correction decreases equivocal studies compared to prone imaging. ${ }^{10}$ However, in the same study utilizing Tc-99mbased rest and stress imaging with attenuation correction, prone imaging, and prone and supine imaging without attenuation correction, prone imaging significantly reduced equivocal studies. ${ }^{10}$ Indeed, even with a state-of-the-art SPECT-CT unit employing novel collimation and iterative reconstruction techniques, the addition of prone imaging may reduce the incidence of apical artifacts. ${ }^{11}$

Therefore, combining supine and prone imaging routinely in those labs that do not yet employ attenuation correction fits well with an overall approach to improving quality. The technique represents a method that can increase diagnostic accuracy without increasing radiation exposure or incurring the expense of new equipment. What about the extra time that an additional, prone, acquisition requires? With MPI volume falling nationally, ${ }^{14}$ it is hard to argue that most labs do not have the $<10$ additional minutes that another acquisition would require. Balancing this time vs measurable 
increases in test accuracy thereby reducing the possible need to proceed with invasive evaluation makes it hard to argue against performing combined supine and prone cardiac SPECT in labs without attenuation correction.

\section{Disclosure}

None.

\section{References}

1. Taasan V, Wokhlu A, Taasan MV, Dusaj RS, Mehta A, Kraft S, et al. Comparative accuracy of supine-only and combined supineprone myocardial perfusion imaging in men. J Nucl Cardiol 2015. doi:10.1007/s12350-015-0358-2.

2. Segall GM, Davis MJ, Goris ML. Improved specificity of prone versus supine thallium SPECT imaging. Clin Nucl Med 1988;13: 915-6.

3. Segall GM, Davis MJ. Prone versus supine thallium myocardial SPECT: A method to decrease artifactual inferior wall defects. J Nucl Med 1989;30:548-55.

4. Perault C, Loboguerro A, Lien JC, Wampach H, Gibold C, Ouzan $\mathrm{J}$, et al. Quantitative comparison of prone and supine myocardial SPECT images. Clin Nucl Med 1995;20:678-84.

5. Hayes SW, De Lorenzo A, Hachamovitch R, Dhar SC, Cohen I, Hsu P, et al. Prognostic implications of combined prone and supine acquisitions in patients with equivocal or abnormal supine myocardial perfusion SPECT. J Nucl Med 2003;44:1633-40.

6. Nishina J, Slomka PJ, Abidov A, Yoda S, Akincioglu C, Kang X, et al. Combined supine and prone quantitative myocardial perfusion SPECT: Method development and clinical validation in patients with no known coronary artery disease. J Nucl Med 2006; 47:51-8.

7. Slomka PJ, Nishina H, Abidov A, Hayes SW, Friedman JD, Berman DS, et al. Combined quantitative supine-prone myocardial perfusion SPECT improves detection of coronary artery disease and normalcy rates in women. J Nucl Cardiol 2007;14:44-52.
8. Berman DS, Kang XP, Nishina H, Slomka PJ, Shaw LJ, Hayes $\mathrm{SW}$, et al. Diagnostic accuracy of gated Tc-99m sestamibi stress myocardial perfusion SPECT with combined supine and prone acquisitions to detect coronary artery disease in obese and nonobese patients. J Nucl Cardiol 2006;13:191-201.

9. Arsanjani R, Hayes SW, Fish M, Shalev A, Nakanishi R, Thomson $\mathrm{L}$, et al. Two-position supine/prone myocardial perfusion SPECT (MPS) imaging improves visual inter-observer correlation and agreement. J Nucl Cardiol 2014;21:703-11.

10. Malkemeker D, Brenner R, Martin WH, Sampson UK, Feurer ID, Kronenberg MW, et al. CT-based attenuation correction versus prone imaging to decrease equivocal interpretation of rest/stress Tc-99m tetrofosmin SPECT MPI. J Nucl Cardiol 2007;14: 314-23.

11. Takamura T, Horiguchi Y, Kanna M, Matsushita H, Sudo Y, Kikuchi S, et al. Validation of prone myocardial perfusion SPECT with a variable-focus collimator versus supine myocardial perfusion SPECT with or without computed tomography-derived attenuation correction. Ann Nucl Med 2015;29:890-6.

12. Cerqueira MD, Allman KC, Ficaro EP, Hansen CL, Nichols KJ, Thompson RC, et al. Recommendations for reducing radiation exposure in myocardial perfusion imaging. J Nucl Cardiol 2010; 17:709-18.

13. Reyes E, Wiener S, Underwood SR, European Council of Nuclear Cardiology. Myocardial perfusion scintigraphy in Europe 2007: A survey of the European Council of Nuclear. Cardiology 2012;39:160-4.

14. 2013 American Society of Nuclear Cardiology/MedAxiom Nuclear Survey. J Nucl Cardiol 2014;21:S5-88.

15. DePuey EG, Bommireddipalli S, Clark J, Thompson L, Srour Y. Wide beam reconstruction "quarter-time" gated myocardial perfusion SPECT functional imaging: A comparison to "full-time", ordered subset expectation maximum. J Nucl Cardiol 2009;16: 736-52.

16. Chang SM, Nabi F, Xu J, Mahmarian JJ. Normal stress-only versus standard stress/rest myocardial perfusion imaging: Similar patient mortality with reduced radiation exposure. J Am Coll Cardiol 2010;55:221-30.

17. Mahmarian JJ. Stress only myocardial perfusion imaging: Is it time for a change? J Nucl Cardiol 2010;17:529-35. 